\title{
Avaliação sensorial da carne de cordeiros machos e fêmeas Texel $x$ Corriedale terminados em diferentes sistemas
}

\section{Marlice Salete Bonacina ${ }^{1}$, Maria Teresa Moreira Osório ${ }^{2}$, José Carlos da Silveira Osório², Gladis Ferreira Corrêa ${ }^{3}$, Juliano Hideo Hashimoto ${ }^{1}$, Rosilene Inês Lehmen ${ }^{4}$}

\footnotetext{
1 Pós-Graduando em Zootecnia - FAEM/UFPeL.

2 Departamento de Zootecnia-FAEM/UFPeL.

3 Universidade Federal do Pampa.

${ }^{4}$ Curso de Agronomia - FAEM/UFPeL.
}

RESUMO - O objetivo neste trabalho foi avaliar o efeito do sexo e de três sistemas de terminação nas características sensoriais da carne de cordeiros Texel × Corriedale e na aceitação da carne pelo consumidor. Foram utilizados 90 animais, 45 cordeiros machos não-castrados e 45 fêmeas mantidos em pastagem até o desmame (70 dias) e terminados em três sistemas de produção: pastagem; pastagem ao pé da mãe; e pastagem com suplementação (casca de soja em nível correspondente a $1 \%$ do peso vivo dos cordeiros). Após o abate, as carcaças foram armazenadas em câmara fria, com ar forçado, a $1^{\circ} \mathrm{C}$, durante 24 horas, para retirada do músculo longissimus dorsi, que foi congelado a $-18^{\circ} \mathrm{C}$ para análise sensorial. A caracterização sensorial da carne foi realizada por meio da análise descritiva quantitativa: 22 termos descritivos foram desenvolvidos por uma equipe de julgadores selecionados, que geraram também a definição de cada termo e as amostras-referência. Foi realizado um teste de aceitação utilizando escala hedônica híbrida de nove pontos. A carne dos machos e dos animais terminados em pastagem ao pé da mãe caracterizou-se pelo odor e sabor residual mais suaves de carne ovina e gordura, menor maciez e maior mastigabilidade em comparação à das fêmeas e dos animais terminados nos demais sistemas. As carnes dos cordeiros terminados nos sistemas de pastagem e de pastagem com suplementação são semelhantes quanto aos aspectos sensoriais. A carne é igualmente aceita pelos consumidores, independentemente do sexo e do sistema de terminação, apresentando boa aceitação.

Palavras-chave: análise descritiva quantitativa, ovinos, qualidade, sexo

\section{Sensory evaluation of meat lambs from males and femeles Texel $x$ Corriedale finished in different systems}

\begin{abstract}
The objective of this work was to evaluate the effect of sex and of three finishing systems on sensory traits of Texel $\times$ Corriedale lamb meat an on the consumer acceptance of the meat. It was used 90 animals, 45 non-castrated male lambs and 45 females kept on pasture until weaning (70 days of age) and finished in three production systems: pasture, pasture with mother, and pasture with supplementation (soybean hull corresponding to $1 \%$ of life weight of the lambs). After slaughter, carcasses were stored in cold chamber, with forced air at $1^{\circ} \mathrm{C}$ until reaching 24 hours for removal of longissimus dorsi muscle which had been frozen at $-18^{\circ} \mathrm{C}$ for sensory analysis. Meat sensorial characterization was performed by quantitative descriptive analyses: 22 descriptive terms were developed by a team of selected judges who also created the definition for each term and the reference-samples. Acceptability test was performed by using a nine-point hybrid hedonic scale. Meat of male animals and of animals finished in pasture with the mother was characterized by the flavor and softer residual taste sheep meat and fat, less softness and higher chewiness in comparison to the meat of female and of animals finished in the other systems. Meat of lambs finished in pasture systems and pasture with supplementation were similar regarding to sensory aspects. Meat was equally accepted by the consumers, regardless of sex and finishing system, presenting good acceptability.
\end{abstract}

Key Words: quality, quantitative-descriptive analysis method, sex, sheep

\section{Introdução}

A última década foi caracterizada por importantes mudanças nos hábitos alimentares dos consumidores de carne (Hoffman et al., 2003). A busca por alimentos mais saudáveis e a maior exigência em relação à qualidade dos produtos direcionaram parte do nicho de mercado a consumir carnes de melhor qualidade nutricional e sensorial (Costa et al., 2008).

Entre as carnes vermelhas consumidas no Brasil, a ovina tem se destacado pela melhoria nas condições de abate, com fiscalização oficial e maior disponibilidade de 
categorias jovens, que resultam em carcaças de melhor qualidade (Sobrinho et al., 2005). Segundo Costa et al. (2008), para obtenção de carne de qualidade, a produção de ovinos deve ser planejada visando à obtenção de carnes com níveis aceitáveis de gordura, visto que promove melhor qualidade sensorial do produto. De acordo com esses autores, o genótipo, o peso de abate, o sexo, o sistema de produção e principalmente a dieta devem ser considerados no planejamento produtivo, priorizando-se a qualidade nutricional e sensorial da carne, como forma de atender às novas perspectivas do mercado, sem deixar de se estabelecer adequada relação custo/benefício.

A alimentação dos animais pode determinar das características sensoriais da carne. Segundo Cañeque et al. (1989), os concentrados, por exemplo, promovem aumento da suculência da carne de cordeiros e, como alteram a composição em ácidos graxos da gordura, permitem modificar o sabor e o odor.

Por outro lado, o efeito do sexo do animal, a questão é discutível. Touralle (1991) e Gularte et al. (2000) verificaram que a carne das fêmeas é mais macia que a dos machos quando o animal tem a mesma idade, porém Sañudo (1991), em pesquisa com animais jovens, não encontrou diferença na maciez da carne de machos e fêmeas.

Os atributos sensoriais são importantes para o consumidor, pois, na avaliação da qualidade de carne, termos como maciez, suculência e cor são destacados (Pearson \& Dutson, 1994). A avaliação sensorial da carne depende de fatores como tipo de julgador, método de cozimento, forma de preparação das amostras e tipo de músculo utilizado (Resurreccion, 2003).

Em face disso, realizou-se este trabalho com os objetivos de investigar o efeito do sexo e de diferentes sistemas de terminação de cordeiros Texel $\times$ Corriedale nas características sensoriais da carne e avaliar a aceitação desse produto pelo consumidor.

\section{Material e Métodos}

O trabalho experimental, em sua fase de campo foi realizado entre os meses de outubro de 2006 a maio de 2007 em uma propriedade rural situada no município de Arroio Grande no estado do Rio Grande do Sul, localizada entre os paralelos $32^{\circ} 14^{\prime} 16^{\prime \prime}$ sul e $53^{\circ} 05^{\prime} 13^{\prime \prime}$ oeste, onde o clima é subtropical, com estações bem definidas e temperatura média anual de $17,5^{\circ} \mathrm{C}$. A fase laboratorial foi desenvolvida no Laboratório de Análise Instrumental e Sensorial do Departamento de Zootecnia da Faculdade de Agronomia Eliseu Maciel da Universidade Federal de Pelotas.
Foram utilizados 90 animais: 45 cordeiros machos não-castrados e 45 fêmeas. Os animais foram mantidos em pastagem até o desmame (70 dias) e terminados em três sistemas de produção: pastagem, pastagem ao pé da mãe e pastagem com suplementação (casca de soja em nível correspondente a $1 \%$ do peso vivo). Em cada sistema de produção, foram alocados 15 fêmeas e 15 machos nãocastrados, porém, durante o experimento, duas fêmeas do sistema de pastagem com suplementação morreram, permanecendo 28 animais.

Os cordeiros criados em pastagem ao pé da mãe foram mantidos em um piquete com predominância de Eryngium horridum (31,96\%), Pennisetum clandestinum $(22,86 \%)$, Baccharis trimera (18,05\%) e Piptochaetium montevidense (6,02\%), enquanto no sistema de terminação em pastagem a vegetação predominante foi composta de Cynodon dactylon (32,63\%), Lolium multiflorum (10,31\%), Eryngium horridum (3,51\%) e Avena strigosa (1,96\%). Já os cordeiros terminados em pastagem com suplementação alimentaram-se de vegetação composta predominantemente de Pennisetum clandestinum (22,52\%), Eryngium horridum (7,43\%), Lolium multiflorum (7,24\%) e Cynodon dactylon (5,34\%). A avaliação da pastagem foi realizada pelo método botanal (Tothill et al., 1992).

Os animais foram abatidos conforme as normas do Regulamento da Inspeção Industrial e Sanitária de produtos de Origem Animal - RIISPOA (Brasil, 2000). O critério utilizado para determinação do abate dos cordeiros foi a condição corporal variando na faixa de 2 a 3, seguindo a exigência do mercado naquele momento. A avaliação da condição corporal foi realizada através da palpação ao longo das apófises espinhosas dorsais, lombares e da base da cauda conforme metodologia descrita por Osório et al. (1998). Após o abate dos animais, as carcaças foram armazenadas em câmara fria, com ar forçado, à temperatura de $1^{\circ} \mathrm{C}$, até completar 24 horas post mortem. As carcaças foram então divididas ao meio e o músculo longissimus dorsi retirado e congelado a $-18^{\circ} \mathrm{C}$ para análise sensorial.

Para realização da análise sensorial, o músculo longissimus dorsi foi primeiramente descongelado sob refrigeração a $4^{\circ} \mathrm{C}$, durante 24 horas, enrolado em papel alumínio e assado até atingir temperatura interna de $70^{\circ} \mathrm{C}$. As amostras da carne assada foram cortadas paralelamente às fibras musculares em cubos de $1,5 \mathrm{~cm}$ e servidas a temperatura de aproximadamente $60^{\circ} \mathrm{C}$ (Rota, 2005).

As características sensoriais de cada amostra de carne foram determinadas utilizando-se a Análise Descritiva Quantitativa (ADQ) descrita por Stone \& Sidel (1998), com julgadores treinados. Foi ainda realizado um teste de 
aceitação com consumidores do produto, conforme recomendações de Meilgaard et al. (1987).

Para realização da ADQ, foram recrutados 25 julgadores (Queiroz \& Treptow, 2006), entre os alunos e funcionários da Universidade Federal de Pelotas. Foram realizados testes de reconhecimento de odor e gostos básicos, bem como testes de ordenação utilizando escalas não-estruturadas, para familiarizar os candidatos à técnica de análise sensorial. Depois, para seleção dos julgadores com maior acuidade sensorial, foi aplicado o teste triangular com amostras de carne ovina. A análise dos resultados do teste triangular foi realizada por análise sequencial de Wald (Queiroz \& Treptow, 2006) mediante um sistema de decisão obtido de acordo com as retas de aceitação ( $\mathrm{NA}=\mathrm{H}_{0}+\mathrm{Sn}$ ) e rejeição $\left(\mathrm{RN}=-\mathrm{H}_{1}+\mathrm{Sn}\right)$, calculadas a partir dos parâmetros estatísticos: $\mathrm{P}_{0}=0,45$ (máxima habilidade inaceitável), $\mathrm{P}_{1}=0,70$ (mínima habilidade aceitável), $\alpha=0,05$ (probabilidade de acertar sem acuidade), $\beta=0,05$ (probabilidade de rejeitar com acuidade). As equações 1, 2 e 3 fornecem os valores da inclinação da reta (S) e as variáveis independentes da reta de aceitação $\left(\mathrm{H}_{0}\right)$ e rejeição $\left(\mathrm{H}_{1}\right)$.

$$
S=\frac{\log \left(\frac{1-P_{0}}{1-P_{1}}\right)}{\log \frac{P_{1}}{P_{0}}\left(\frac{1-P_{0}}{1-P_{1}}\right)} \quad H_{0}=\frac{\log \left(\frac{1-\alpha}{\beta}\right)}{\log \frac{P_{1}}{P_{0}}\left(\frac{1-P_{0}}{1-P_{1}}\right)} \quad H_{1}=\frac{\log \left(\frac{1-\beta}{\alpha}\right)}{\log \frac{P_{1}}{P_{0}}\left(\frac{1-P_{0}}{1-P_{1}}\right)}
$$

Traçam-se as retas definidas por essas equações em um eixo de coordenadas, plotando-se na ordenada o número de respostas acumuladas e nas abscissas o número de provas (Figura 1).

O desenvolvimento da terminologia descritiva das amostras de carne foi feito utilizando-se o método de rede, descrito por Kelly e citado por Moskowitz (1983). Em cabines individuais de avaliação sensorial, as seis amostras de

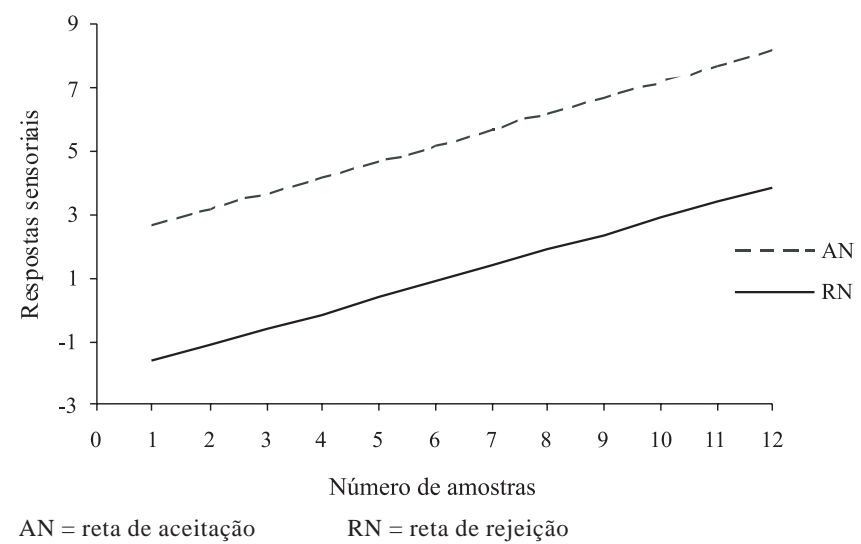

Figura 1 - Representação gráfica da sequencial de Wald (Queiroz \& Treptow, 2006) carne foram apresentadas aos pares, envolvendo todas as combinações possíveis, a 12 julgadores previamente selecionados. Os julgadores foram solicitados a descrever as similaridades e diferenças entre as amostras de cada par com relação aos atributos sensoriais.

Depois que cada julgador gerou seus próprios termos, foi realizada uma discussão em grupo, sob a supervisão de um moderador, com o objetivo de agrupar termos semelhantes e eliminar aqueles que não eram percebidos pela maioria dos julgadores. Em seguida elaborou-se uma ficha de avaliação contendo os termos escolhidos em consenso pela equipe e a escala não-estruturada de $9 \mathrm{~cm}$, ancorada nos extremos, à esquerda pelo termo "pouco" e a direita "muito" para cada atributo listado. Nessa etapa foi também elaborada uma lista de definições dos termos descritivos com as respectivas referências, para melhor uniformizar a avaliação dos julgadores (Tabela 1).

$\mathrm{O}$ treinamento dos julgadores quanto à terminologia e sua quantificação foi realizado utilizando testes de ordenação e uso de escala não-estruturada, conforme Szczesniak (1979) e Muñoz (1999). Durante o treinamento, os julgadores foram solicitados a avaliar nas amostras de carne ovina a intensidade dos atributos sensoriais, sendo permitido consultar a qualquer momento a lista de definições e as referências. O treinamento foi encerrado após verificar as habilidades individuais dos julgadores, sendo selecionados os que apresentaram maior poder discriminatório e reprodutibilidade dos resultados.

A caracterização sensorial das seis amostras de carne ovina foi realizada por nove julgadores resultantes do treinamento. Foi utilizado o delineamento inteiramente casualizado com três repetições para cada amostra por julgador, usando-se a técnica de apresentação monádica. A intensidade de cada descritor foi medida nas amostras utilizando a escala não-estruturada de $9 \mathrm{~cm}$.

A aceitação das seis amostras de carne foi avaliada por 84 consumidores, 38 mulheres e 46 homens, com idade na faixa de 19 a 68 anos. A frequência de consumo de carne ovina pelos consumidores que participaram do teste variou entre pelo menos uma vez na semana até uma vez ao ano.

O teste foi realizado em laboratório, sob condições controladas. As amostras de carne foram conservadas em salmoura a $10 \%$ a proporção de $1: 1$ de peso, a temperatura de $5^{\circ} \mathrm{C}$, por uma hora (Siqueira et al., 2002). Depois, a carne foi enrolada em papel-alumínio e assada até atingir temperatura interna de $70^{\circ} \mathrm{C}$, sendo então cortada paralelamente às fibras musculares em cubos de $1,5 \mathrm{~cm}$ e servidas a $60^{\circ} \mathrm{C}$. 
Tabela 1 - Lista de definição dos termos e respectivas referências

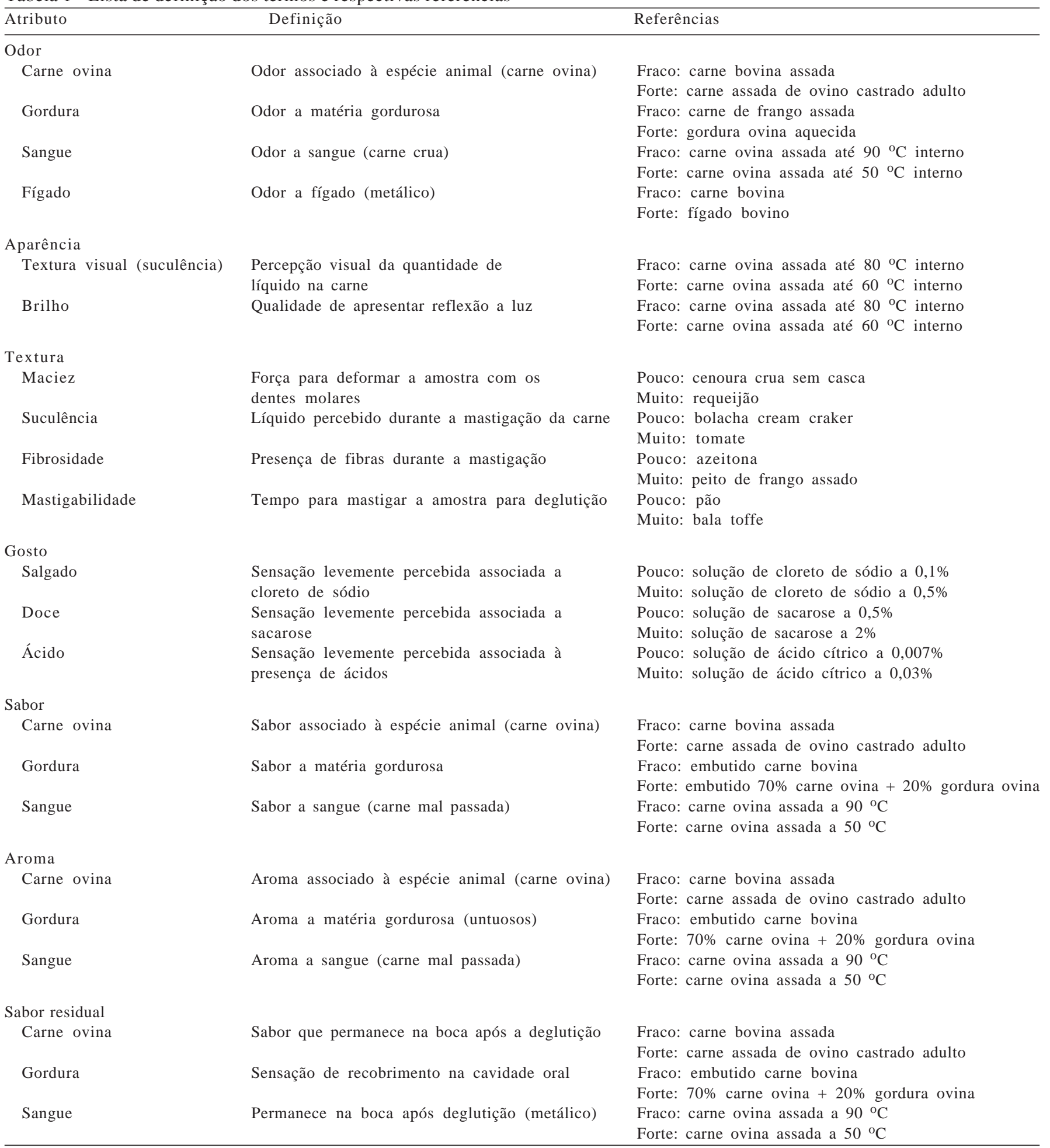

Os julgadores receberam as amostras de forma monádica, codificadas com número de três dígitos aleatórios, sendo a ordem de apresentação de forma balanceada. Para avaliar a aceitação das amostras de carne, os julgadores utilizaram uma escala hedônica híbrida de $9 \mathrm{~cm}$, ancorada na região central e nos extremos com anotações verbais: desgostei muitíssimo à esquerda; gostei muitíssimo à direita; e nem gostei/nem desgostei ao centro.

Para análise dos dados, foi utilizando o software Estatística 6.0 (Statsoft, 2001). Os resultados da análise descritiva quantitativa foram submetidos à análise de variância de duas fontes de variação amostras e repetição para cada termo e julgador. Foram computados para cada 
julgador em cada termo os valores de $\mathrm{F}_{\text {amostra }}$ significativos para $\mathrm{p}<0,5$ e $\mathrm{F}_{\text {repetição }}$ não-significativos para $\mathrm{p}>0,05$, para demonstrar poder discriminativo e reprodutibilidade dos julgamentos, respectivamente, conforme metodologia proposta por Damásio \& Costell (1991). Aplicou-se o teste de Tukey a 5\% de significância para a comparação entre as médias. Os resultados do teste de aceitação foram analisados por meio da análise de variância e teste Tukey.

Tabela 2 - Valores p do desempenho da equipe de julgadores em relação a seu poder discriminatório ( $\left.\mathrm{p}_{\text {amostra }}\right)$ e repetibilidade $\left(\mathrm{p}_{\text {repetição }}\right)$

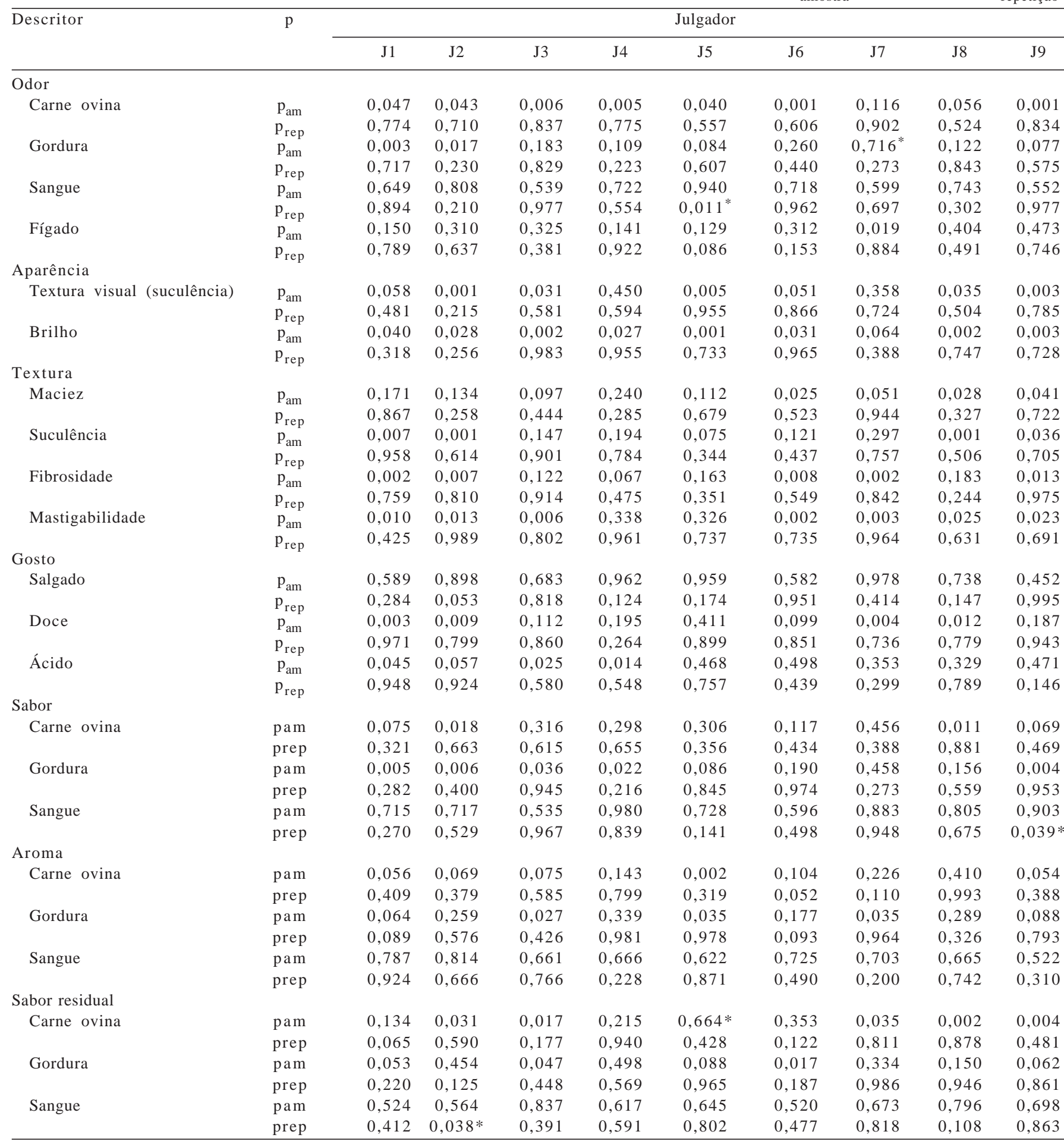

Para avaliação sensorial, a equipe de julgadores utilizou uma escala de $9 \mathrm{~cm}$.

* valores não atendendo aos valores especificados para $\mathrm{p}_{\text {amostra }}$ e $\mathrm{p}_{\text {repeticão. }}$

$\mathrm{p}_{\text {amostra }}<0,5$ significa que o julgador discriminou as amostras para o termo sensorial avaliado.

$\mathrm{P}_{\text {repetição }}>0,05$ significa que o julgador não apresentou repetibilidade para o termo sensorial avaliado. 


\section{Resultados e Discussão}

De modo geral, o desempenho dos julgadores foi satisfatório (Tabela 2), pois, entre os nove julgadores treinados, apenas os julgadores $\mathrm{J}_{5} \mathrm{e}_{7}$ apresentaram menor capacidade discriminatória, uma vez que não conseguiram diferenciar as amostras quanto ao sabor residual de carne ovina e odor a gordura, respectivamente. A equipe de julgadores não discriminou as amostras quanto ao gosto salgado e odor, sabor, aroma, sabor residual de sangue, indicando que as amostras não diferiram entre si quanto a esses aspectos.

Além disso, foi verificado consenso da equipe, já que apenas os julgadores $\left(\mathrm{J}_{2}, \mathrm{~J}_{5}\right.$ e $\left.\mathrm{J}_{9}\right)$ apresentaram problema de repetibilidade para o termo sangue, fato que pode ser devido à falta de homogeneidade das repetições fornecidas aos julgadores, provavelmente por problemas durante a etapa em que as amostras foram assadas.
As características sensoriais avaliadas na carne pela equipe de julgadores não sofreram influência da interação entre sexo e sistema de terminação dos cordeiros $(\mathrm{P}>0,05)$; no entanto, os fatores isolados tiveram efeito significativo $(p<0,05)$ em alguns dos termos sensoriais (Tabela 3$)$, expressos pelos valores médios atribuídos pela equipe de julgadores a cada descritor para cada amostra de carne de cordeiro avaliada.

As respostas sensoriais obtidas, considerando os sistemas de terminação dos cordeiros, comprovam que a carne dos animais terminados em pastagem ao pé da mãe foi a que mais se diferiu dos demais sistemas, por apresentar menor intensidade ao odor e sabor a carne ovina, bem como menor intensidade de brilho em relação à aparência, além de menor maciez e maior fibrosidade e mastigabilidade. Apresentou ainda maior intensidade dos gostos doce e ácido e menor sabor e sabor residual a gordura.

Na comparação entre sexos, a carne das fêmeas distinguiu-se da carne dos machos por apresentar odor,

Tabela 3 - Média dos valores atribuídos pelos julgadores para as amostras de carne

\begin{tabular}{|c|c|c|c|c|c|}
\hline \multirow[b]{2}{*}{ Descritor } & \multicolumn{3}{|c|}{ Sistema de terminação } & \multicolumn{2}{|c|}{ Sexo } \\
\hline & Pastagem & Pastagem ao pé da mãe & Pastagem com suplemento & Fêmea & Macho \\
\hline \multicolumn{6}{|l|}{ Odor } \\
\hline Carne ovina & $4,85 b$ & $4,60 \mathrm{a}$ & $4,85 b$ & 4,91B & $4,63 \mathrm{~A}$ \\
\hline Gordura & $4,28 \mathrm{a}$ & $4,21 \mathrm{a}$ & $4,29 a$ & $4,41 \mathrm{~B}$ & $4,11 \mathrm{~A}$ \\
\hline Sangue & $2,43 a$ & $2,53 a$ & $2,50 a$ & $2,46 \mathrm{~A}$ & $2,52 \mathrm{~A}$ \\
\hline Fígado & $2,11 \mathrm{a}$ & $2,13 \mathrm{a}$ & $2,09 a$ & $2,18 \mathrm{~B}$ & $2,09 \mathrm{~A}$ \\
\hline \multicolumn{6}{|l|}{ Aparência } \\
\hline Textura visual (suculência) & $4,66 \mathrm{a}$ & $4,55 a$ & $4,54 a$ & $4,96 \mathrm{~B}$ & $4,21 \mathrm{~A}$ \\
\hline Brilho & $4,25 b$ & $3,89 a$ & $4,00 \mathrm{a}$ & $4,40 \mathrm{~B}$ & $3,69 \mathrm{~A}$ \\
\hline \multicolumn{6}{|l|}{ Textura } \\
\hline Maciez & $5,25 b$ & $5,02 \mathrm{a}$ & $5,26 b$ & $5,38 \mathrm{~B}$ & $4,98 \mathrm{~A}$ \\
\hline Suculência & $4,62 \mathrm{a}$ & $4,59 a$ & $4,56 a$ & $4,76 \mathrm{~B}$ & $4,42 \mathrm{~A}$ \\
\hline Fibrosidade & $2,62 \mathrm{a}$ & $3,32 b$ & $2,79 a$ & $2,91 \mathrm{~A}$ & $2,92 \mathrm{~A}$ \\
\hline Mastigabilidade & $2,50 a$ & $3,08 b$ & $2,72 \mathrm{a}$ & $2,61 \mathrm{~A}$ & $2,93 \mathrm{~B}$ \\
\hline \multicolumn{6}{|l|}{ Gostos } \\
\hline Salgado & $2,40 \mathrm{a}$ & $2,36 a$ & $2,36 a$ & $2,40 \mathrm{~A}$ & $2,35 \mathrm{~A}$ \\
\hline Doce & $1,82 \mathrm{a}$ & $2,37 c$ & $2,08 b$ & $2,11 \mathrm{~A}$ & $2,07 \mathrm{~A}$ \\
\hline Ácido & $1,97 \mathrm{a}$ & $2,28 \mathrm{c}$ & $2,07 b$ & $2,08 \mathrm{~A}$ & $2,13 \mathrm{~A}$ \\
\hline \multicolumn{6}{|l|}{ Sabor } \\
\hline Carne ovina & $4,73 a$ & $4,66 a$ & $4,65 a$ & $4,76 \mathrm{~B}$ & $4,60 \mathrm{~A}$ \\
\hline Gordura & $3,96 b$ & $3,66 a$ & $3,76 a b$ & $4,01 \mathrm{~B}$ & $3,57 \mathrm{~A}$ \\
\hline Sangue & $2,20 \mathrm{a}$ & $2,24 \mathrm{a}$ & $2,30 \mathrm{a}$ & $2,23 \mathrm{~A}$ & $2,27 \mathrm{~A}$ \\
\hline \multicolumn{6}{|l|}{ Aroma } \\
\hline Carne ovina & $4,36 a$ & $4,29 a$ & $4,31 \mathrm{a}$ & $4,41 B$ & $4,20 \mathrm{~A}$ \\
\hline Gordura & $3,52 \mathrm{a}$ & $3,37 \mathrm{a}$ & $3,42 \mathrm{a}$ & $3,54 \mathrm{~B}$ & $3,33 \mathrm{~A}$ \\
\hline Sangue & $2,25 a$ & $2,31 \mathrm{a}$ & $2,28 \mathrm{a}$ & $2,29 \mathrm{~A}$ & $2,28 \mathrm{~A}$ \\
\hline \multicolumn{6}{|l|}{ Sabor residual } \\
\hline Carne ovina & $4,18 b$ & $3,73 a$ & $3,98 b$ & $4,08 \mathrm{~B}$ & $3,85 \mathrm{~A}$ \\
\hline Gordura & $3,37 b$ & $2,96 \mathrm{a}$ & $3,08 a$ & $3,23 \mathrm{~B}$ & $3,09 \mathrm{~A}$ \\
\hline Sangue & $2,10 \mathrm{a}$ & $2,16 a$ & $2,22 \mathrm{a}$ & $2,19 \mathrm{~A}$ & $2,13 \mathrm{~A}$ \\
\hline
\end{tabular}

Médias seguidas de letras minúsculas diferentes na mesma linha diferem $(\mathrm{p}<0,05)$ entre sistemas de terminação.

Médias seguidas de letras maiúsculas diferentes na mesma linha diferem $(p<0,05)$ entre sexos. 
sabor, aroma e sabor residual a carne ovina e gordura mais intenso, além de maior intensidade ao odor a fígado e maior textura visual (suculência) e brilho, bem como maior maciez e suculência.

Parte desses resultados pode ser explicada pelo fato de que a carne dos machos e a dos animais terminados em pastagem ao pé da mãe, ter apresentaram menor teor de gordura (Bonacina et al., 2011), pois, conforme Kemp et al. (1981), há relação entre os níveis quantitativos de gordura e os atributos sensoriais da carne, de modo que, quanto maior o teor de gordura, maior a maciez e suculência da carne. Fisher et al. (2000) salientam que as diferenças sensoriais percebidas na carne ovina por julgadores treinados são, em grande parte, resultado da variação do teor de gordura presente na carne. Segundo Osório et al. (2009), as características da carne ovina estão diretamente relacionadas ao teor de gordura presente no músculo.

Madruga et al. (2005) estudaram a qualidade da carne de cordeiros Santa Inês terminados com diferentes dietas e também verificaram que a carne com maior conteúdo de gordura possui odor a carne ovina mais intenso, bem como maior maciez e suculência. Sañudo et al. (2000) também constataram carnes mais macias e com maior intensidade de sabor em decorrência dos maiores escores de gordura. De acordo com Lawrie (2005), a gordura tende a diluir o tecido conjuntivo dos elementos da fibra muscular na qual está depositada, proporcionando maior maciez.

A menor maciez da carne de machos e animais terminados em pastagem ao pé da mãe confirmou os resultados obtidos na análise instrumental de maciez (Bonacina et al., 2011), indicando que os julgadores foram capazes de identificar pequenas diferenças na resistência ao corte da carne. Gonçalves et al. (2004) também observaram que a carne das fêmeas foi significativamente mais macia que a dos machos não-castrados. De acordo com Bonagurio et al. (2003), os machos, além de menor quantidade de gordura na carne em comparação às fêmeas, normalmente apresentam constituição muscular mais densa, o que resulta em carne menos macia.

No entanto, Tajeda et al. (2008) não notaram diferença significativa nos atributos sensoriais quando compararam o sexo dos cordeiros, porém a intensidade do odor, sabor, maciez e suculência foi maior nas fêmeas.

A suculência da carne segue a mesma tendência da maciez, quando considerado o sexo, concordando com os estudos de Kemp et al. (1981). Os resultados obtidos devem-se, provavelmente, ao maior conteúdo de gordura encontrado na carne das fêmeas (Bonacina et al., 2011), pois, segundo Pardi et al. (1996), a gordura tem efeito estimulante sobre a salivação, prolongando a sensação de suculência ao longo da mastigação.

A presença de concentrado na dieta dos cordeiros provocou pouca alteração nas características sensoriais da carne, ocasionando diferença significativa $(\mathrm{p}<0,05)$ apenas para o gosto doce, ácido e sabor residual de gordura em comparação à carne dos cordeiros terminados em pastagem. Ferrão et al. (2009) verificaram que o uso de dietas com concentrado não promoveu diferenças significativas perceptíveis pelo painel sensorial nos parâmetros de sabor, maciez e suculência na carne de cordeiros Santa Inês.

Em estudo sobre as propriedades sensoriais da carne ovina no Nordeste brasileiro, Zapata et al. (2000), utilizando mestiços Somalis Brasileira $\times$ Crioula e Santa Inês $\times$ Crioula submetidos a duas dietas (feno e feno $+20 \%$ de concentrado) concluíram que não houve efeito da dieta sobre os parâmetros sensoriais avaliados nas carnes de pernil. No entanto, segundo Cañeque et al. (1989), os concentrados promovem aumento da suculência da carne de cordeiros por alterar a composição em ácidos graxos da gordura permitindo modificar o sabor e o odor.

A maior intensidade do gosto ácido na carne de cordeiros sob suplementação pode ser explicada pela digestibilidade da FDN da casca de soja, que é bastante alta, proporcionando elevada produção de ácidos graxos voláteis no rúmen, em razão da excelente fermentabilidade da fibra no rúmen (Bach et al., 1999) e dos benefícios decorrentes da digestão da fibra da dieta total sobre o $\mathrm{pH}$ ruminal (Ludden et al., 1995; Gomes, 1998). Além disso, nos ruminantes, parte dos ácidos graxos insaturados provenientes da dieta é saturada por meio de um processo de biohidrogenação no ambiente ruminal como forma de neutralizar o efeito tóxico desses ácidos graxos aos microrganismos ruminais. Como resultado desse processo, a classe dos ácidos graxos saturados é absorvida e incorporada ao nível de tecido muscular (Ponnampalam et al., 2001). Petrova et al. (1994) salientaram que uma dieta à base de concentrado tem elevada quantidade de carboidratos rapidamente degradáveis no rúmen, fato que pode ter intensificado o gosto doce na carne dos cordeiros sob suplementação.

Na carne dos cordeiros (Tabela 3) terminados em pastagem ao pé da mãe, o gosto doce provavelmente é decorrente da presença de carboidratos no leite, enquanto o gosto ácido pode ser oriundo da quantidade de ácidos graxos saturados nos lipídios do leite, pois, de acordo com Todaro et al. (2006), a dieta exclusiva de leite induziu maior concentração de ácidos graxos saturados na carne de cabritos, possivelmente em virtude da quantidade de saturados nos lipídios do leite. 
Em análise dos resultados do teste de aceitação, não houve interação entre o sexo e o sistema de terminação dos cordeiros ( $>0,05)$, assim como não foi observado efeito significativo dos fatores isolados (sexo e sistema de terminações) sobre a aceitação da carne de cordeiro, cujo valor médio foi de 6,6, pelos julgadores, variando na escala hedônica entre os termos "gostei ligeiramente" a "gostei moderadamente". Zapata et al. (2000), estudando a qualidade da carne ovina do Nordeste brasileiro, realizaram análise sensorial utilizando escala hedônica de 9 pontos com julgadores não treinados e obtiveram pontuação média de 7,1 para a aceitação da carne.

Embora a equipe treinada de julgadores tenha identificado diferenças nas características sensoriais das carnes de cordeiros, as amostras foram igualmente aceitas pelos consumidores e isso pode estar relacionado ao tipo de julgador, pois a equipe treinada pode ter apresentado grande sensibilidade nas respostas sensoriais, ou ainda devido ao fato de se terem utilizado alguns julgadores para o teste de aceitação que não consomem carne com frequência (apenas uma ou duas vezes ao ano), ficando assim pouco familiarizado com o produto. Esse resultado confirma a observação realizada por Font I Furnols et al. (2006), que estudaram a aceitabilidade da carne de cordeiros por consumidores de diferentes países europeus e concluíram que um dos fatores que influem na aceitabilidade da carne é o hábito de consumo.

Além disso, conforme Font I Furnols et al. (2009), a consideração dos consumidores como um todo pode induzir a erros, pois, quando os dados são analisados com mais cuidado, podem ser detectados diferentes nichos de consumidores. Esses autores estudaram, entre consumidores europeus, a aceitabilidade da carne de cordeiros alimentados a pasto, concentrado ou combinação dos dois sistemas e verificaram que, em geral, os consumidores preferem carne de cordeiros alimentados com concentrado ou mistura de concentrado e pastagem. Alguns consumidores apresentam maior aceitabilidade da carne de cordeiros alimentados a pasto em comparação à de animais terminados em sistema a pasto com concentrado.

Ressalta-se ainda que houve boa aceitação global das amostras, enquanto, na análise descritiva quantitativa, os atributos foram avaliados de forma individual, dessa forma, os consumidores podem ter relacionado os atributos de maior e menor aceitação, assim, o grau de satisfação foi avaliado em função do conjunto, ou seja, alguns julgadores podem ter apreciado o odor e sabor menos intenso a carne ovina e a gordura, enquanto outros preferiram a carne de maior maciez e suculência.

\section{Conclusões}

O sistema de terminação e o sexo dos cordeiros Texel $\times$ Corriedale influencia as características sensoriais da carne. Apesar de uma diferença significativa nos aspectos sensoriais, as amostras são igualmente aceitas pelos consumidores.

\section{Referências}

BACH, A.; YOON, I.K.; STERN, M.D. et al. Effects of type of carbohydrate supplementation to lush pasture on microbial fermentation in continuous culture. Journal of Dairy Science, v.82, p.153-160, 1999.

BONAGURIO, S.; PERÉZ, J. R.O.; FURUSHO GARCIA, I.F. et al. Qualidade da carne de cordeiros Santa Inês e mestiços com Texel abatidos com diferentes pesos. Revista Brasileira de Zootecnia, v.32, n.6, p.1981-1991, 2003.

BONACINA, M.S.; OSÓRIO, M.T.M.; OSÓRIO, J.C.S. et al. Influência do sexo e do sistema de terminação de cordeiros Texel x Corriedale na qualidade da carcaça e da carne. Revista Brasileira de Zootecnia, v.40, n.6, p.1242-1249, 2011.

BRASIL. Ministério da Agricultura. Instrução Normativa $n^{\circ}$. 3, de 07 de janeiro de 2000. Regulamento técnico de métodos de insensibilização para o abate humanitário de animais de açougue. S.D.A./M.A.A. Diário Oficial da União, Brasília, p.14-16, 24 de janeiro de 2000, Seção I.

CAÑEQUE, V.; HUILdOBRO, F.R.; DOLZ, J.F. et al. Producción de carne de cordero. Madrid: Ministerio de Agricultura Pesca y Alimentacion, 1989. 520p.

COSTA, R.G.; CARTAXO, F.Q.; SANTOS, N.M. et al. Carne caprina e ovina: composição lipídica e características sensoriais. Revista Brasileira de Saúde e Produção Animal, v.9, n.3, p.497-506, 2008.

DAMASIO, M.H.; COSTELL, E. Análisis sensorial descritivo: generación de descriptors y selección de catadores. Revista Agroquimica de Technologia de Alimentos, v.31, n.2, p.165-178, 1991.

FERRÃO, S.P.B.; BRESSAN, M.C.; OLIVEIRA, R. et al. Características sensoriais da carne de cordeiros da raça Santa Inês submetidos a diferentes dietas. Ciência Agrotecnologia, v.33, n.1, p.185-190, 2009.

FISHER, A.V.; ENSER, M.; RICHARDSON, R.I. et al. Fatty acid composition and eating quality of lamb types derived from four diverse breed production systems. Meat Science, v.55, n.2, p.141-147, 2000.

FONT I FURNOLS, M.F.; SAN JULIÁN, R.; GUERRERO, L. et al. Acceptability of lamb meat from different producing systems and ageing time to German, Spanish and British consumers. Meat Science, v.72, p.545-554, 2006.

FONT I FURNOLS, M.F.; REOLINE, C.E.; GUERRERO, L. et al. Acceptability of lamb fed on pasture, concentrate or combinations of both systems by European consumers. Meat Science, v.81, p.196-202, 2009.

GOMES, I.P.O. Substituição do milho pela casca de soja em dietas com diferentes proporções de volumoso:concentrado para bovinos em confinamento. 1998. 84f. Tese (Doutorado em Zootecnia) - Universidade Estadual Paulista, Jaboticabal.

GONÇALVES, L.A.G; ZAPATA, J.F.F.; RODRIGUES, M.C.P. et al. Efeitos do sexo e do tempo de maturação sobre a qualidade da carne ovina. Ciência Tecnologia de Alimentos v.24, n.3, p.450-467, 2004. 
GULARTE, M.A.; TREPTOW, R.O.; POUEY, J.L.F. et al. Idade e sexo na maciez da carne de ovinos da raça corriedale Ciência Rural, v.30, n.3, p.485-488, 2000.

KEMP, J.D.; MAHYUDDIN, M.; ELY, D.G. Effect of feeding systems, slaughter weight and sex on organoleptic properties, and fatty acid composition of lamb. Journal of Animal, v.51, n.2, p.321-330, 1981.

HOFFMAN, L.C.; MULLER, M.; CLOETE, S.W.P. et al. Comparison of six crossbred lamb types: sensory, physical and nutritional meat quality characteristics. Meat Science, v.65, p.1265-1274, 2003.

LAWRIE, R.A. Ciência da carne. Trad. Jane Maria Rubensam. 6.ed. Porto Alegre: Artmed, 2005. 384p.

LUDDEN, P.A.; CECAVA, M.J.; HENDRIX, K.S. The value of soybean hulls as a replacement for corn in beef cattle diets formulated with or without added fat. Journal of Animal Science, v.73, p.2706-2711, 1995.

MADRUGA, M.S.; SOUSA, W.H.S.; ROSALES, M.D. et al. Qualidade da carne de cordeiros Santa Inês terminados com diferentes dietas. Revista Brasileira de Zootecnia, v.34, n.1, p.309-315, 2005.

MEILGAARD, M.; CIVILLE, G.V.; CARR, B.T. Sensory evaluation techniques. 2v. Boca Raton: CRC Press, 1987. 387p.

MOSKOWITZ, H.R. Product testing and sensory evaluation of foods: Marketing and R\&D approaches. Westport: Food and Nutrition Press, 1983. 605p.

MUÑOZ, A.M. Analise desriptivo - Desarrollo de descriptores. In: ALMEIDA, T.C.A Avanços em análise sensorial. São Paulo: Livraria Varela, 1999. p.23-34

OSÓRIO, J.C.; OSÓRIO, M.T.M.; JARDIM, P.O. et al. Métodos para avaliação da produção de carne ovina: "in vivo" na carcaça e na carne. Universidade Federal de Pelotas, Pelotas, 1998. $107 \mathrm{p}$.

OSÓRIO, J.C.; OSÓRIO, M.T.M; SAÑUDO,C. Características sensoriais da carne ovina Revista Brasileira de Zootecnia, v.38, p.292-300, 2009.

PARDI, M.C.; SANTOS, I.F.; SOUZA, E.R. Ciência, Higiene e Tecnologia da Carne v.1 Goiânia: CEGRAF-UFG, 1996. $586 \mathrm{p}$.

PEARSON, A.M.; DUTSON, T.R. Quality attributes and their measurement in meat, poultry and fish products. New York: Blackie Academic \& Professional, 1994. 505p.

PETROVA, Y.; BANSKALIEVA, V.; DIMOV, V. Effect of feed on distribution of fatty acids at Sn-2-position in triacylglycerols of different adipose tissues in lambs. Small Ruminant Research, v.13, p.263-267, 1994.

PONNAMPALAM, E.N.; SINCLAIR, A.J.; EGAN, A.R. et al. Efect of diets containing $n-3$ fatty acids on muscle long chain n-3 fatty acid content in lambs fed low- and medium- quality roughage diets. Journal of Animal Science, v.79, p.698-706, 2001.
QUEIROZ, M.I.; TREPTOW, R.O. Análise sensorial para avaliação da qualidade dos alimentos. Rio Grande: Editora FURG - Fundação Universidade Federal do Rio Grande, 2006. 268 .

RESURRECCION, A.V.A. Sensory aspects of consumer choices for meat and meat products. Meat Science, v.66, n.1, p.11-20, 2003.

ROTA, E. Castração e idade de abate sobre a qualidade da carne de ovinos da raça Corriedale. 2005. 78f. Tese (Doutorado em Zootecnia) - Faculdade de Agronomia "Eliseu Maciel" Universidade Federal de Pelotas, Pelotas.

SAÑUDO, C. La calidad organoléptica de la carne com especial referencia a la especie ovina. Fatores que la determinan, metodos de medida y causas de variacion. Zaragoza: Universidade de Zaragoza, 1991. 225p.

SAÑUDO, C.; ALFONSO, M.; SÁNCHEZ, A. et al. Carcass and meat quality in light lambs from different fat classes in the EU carcass classification system. Meat Science, v.56 p.89-94, 2000.

SIQUEIRA, E.R.; ROÇA, R.O.; FERNANDES, S. Características sensoriais da carne de cordeiros das raças Hampshire Dow, Santa Inês e mestiços Bergamacia X Corriedale abatidos com quatro distintos pesos. Revista Brasileira de Zootecnia, v.31, n.3, p.1269-1272, 2002

SOBRINHO, A.G.S.; SILVA, A.M.A.; NETO, S.G. et al. Parâmetros qualitativos da carcaça e da carne de cordeiros submetidos a dois sistemas de formulação de ração. Agropecuária Científica no Semi-árido, n.1 p.31-38, 2005.

STATSOFT, Inc. Statistica (data analysis software system), version 6.0., 2001. Disponível em: <www.statsoft.com>. Acesso em: maio de 2009.

STONE, H.; SIDEL, J.L. Quantitative descriptive analysis: Developments, applications, and the future. Food Techology, v.52, n.8, p.48-52, 1998.

SZCZESNIAK, A. Recent devolopements in solving consumer-oriented texture problems. Food Tecnology, v.33, n.10, p.61-66, 1979.

TAJEDA, J.F.; PENA, R.; ANDRÉS, A.I. Effect of live weight and sex on physico-chemical and sensorial characteristics of Merino lamb meat. Meat Science, v.80, p.1061-1067, 2008.

TODARO, M.; CORRAO, A.; BARONE, C.M.A. et al. Use of weaning concentrate in the feeding of suckling kids: effects on meat quality. Small Ruminant Research, v.66, p. 44-50, 2006.

TOTHILL, J.C.; HARGREAVES, J.N.G.; JONES, R.N. et al. Botanal - A comprehensive samplingand computing procedure for estimating pasture yield and composition. 1. Field sampling. Tropical Agronomy Technical Memorandum, n.7, p.24, 1992.

TOURALLE, C. Qualités organoleptiques des viandes bovine et ovine. Theix: Station de Recherches Sul la Viande/I.N.R.A., 1991. p.32-42.

ZAPATA, J.F.F.; SEABRA, L.M.J.; NOGUEIRA, C.M. et al. Estudo da qualidade da carne ovina do nordeste brasileiro: propriedades físicas e sensoriais. Ciência e Tecnologia de Alimentos, v.20, n.2, p.274-277, 2000. 